\title{
Alinhamento frontal estático do joelho e cargas plantares durante a marcha de adultos jovens assintomáticos
}

Static frontal knee alignment and plantar loads during gait in healthy young adults

\author{
Isabel C.N. Sacco ${ }^{1}$, Francis Trombini-Souza ${ }^{2}$, Ana Paula Ribeiro ${ }^{2}$, Aline A. Gomes ${ }^{2}$, Maria I. Roveri ${ }^{3}$, \\ Dominique R. M. V. Silva ${ }^{3}$, Lina G. Cadamuro ${ }^{3}$, Mariana F. Cagliari ${ }^{3}$, Priscila S. Souza ${ }^{4}$
}

Estudo desenvolvido no LABIMPH - Laboratório de Biomecânica do Movimento e Postura Humana do Curso de Fisioterapia do Fofito/FMUSP Depto. de Fisioterapia, Fonoaudiologia e Terapia Ocupacional da Faculdade de Medicina da Universidade de São Paulo, São Paulo, SP, Brasil

1 Profa. Dra. do Fofito/FMUSP, coordenadora do LABIMPH

2 Mestrandos em Ciências da Reabilitação no LABIMPH do Fofito/FMUSP

3 Graduandas em Fisioterapia no Fofito/FMUSP

4 Fisioterapeuta do LABIMPH do Fofito/FMUSP

\section{ENDEREÇO PARA}

CORRESPONDÊNCIA

Profa. Dra. Isabel de C. N. Sacco

Fofito/FMUSP

Rua Cipotânea 51 Cidade Universitária

05360-160 São Paulo SP e-mail: icnsacco@usp.br

\section{ApresentaÇÃo} out. 2008

ACEITO PARA PUBLICAÇÃO mar. 2009
Resumo: Na prática fisioterapêutica, pressupõe-se que alterações posturais do membro inferior influenciem a biomecânica e função dos demais complexos durante o movimento. No entanto, a literatura sobre a relação entre desalinhamentos estáticos do joelho e possíveis alterações dinâmicas ainda é escassa e inconclusiva. Assim, buscou-se avaliar o efeito do alinhamento frontal estático do joelho sobre a distribuição da pressão plantar durante a marcha. Foram avaliados inicialmente 44 adultos jovens assintomáticos. Por fotogrametria digital, mediu-se o ângulo frontal do joelho, classificado como normal $\left(170^{\circ}\right.$ a $\left.175^{\circ}\right)$, valgo $<170^{\circ}$ e varo $>175^{\circ}$. Dado o baixo número de valgos, foram analisados dois grupos: de joelhos normais $(n=18)$ e de joelhos varos $(n=23)$. A distribuição da pressão plantar foi avaliada durante a marcha em cinco áreas. Os grupos mostraram-se estatistica-mente semelhantes em todas as variáveis cinéticas avaliadas em todas as áreas plantares. Joelhos normais apresentaram significativa correlação com o tempo de contato no antepé lateral e médio-pé; e os varos, correlação com a área e tempo de contato em duas e três áreas plantares, e com a pressão integral no antepé lateral. Os resultados mostraram que o desalinhamento frontal de $3^{\circ}$ do joelho, embora com moderada correlação, não influencia a distribuição de cargas na superfície plantar durante a marcha. Sugere-se pois que a avaliação clínica não se limite à avaliação articular estática do joelho, mas inclua atividades dinâmicas.

Descritores: Articulação do joelho; Biomecânica; Marcha; Postura

ABSTRACT:In physical therapy practice, it is assumed that lower-limb posture changes may influence other complexes' biomechanics or function during movement. However, literature on the relationship between static knee alignment and possible dynamic changes is still scarce and inconclusive. This study assessed the effect of static frontal knee alignment on plantar pressure distribution during gait. At first 44 young asymptomatic adults were evaluated. Front knee angles were measured by digital photogrammetry and classified as normal $\left(170^{\circ}\right.$ to $\left.175^{\circ}\right)$, valgus $<170^{\circ}$, or varus $>175^{\circ}$. Given the low number of valgus found, two groups were analysed: normal knees $(n=18)$ and varus knees $(n=23)$. Plantar pressure distribution was measured during gait in five plantar surface areas. Groups were found to be statistically similar in all kinetic variables assessed in all plantar surface areas. Normal knees showed significant correlation with the contact time at lateral forefoot and midfoot. Varus knees showed correlation with contact area and time at respectively two and three plantar areas, and with full pressure at lateral forefoot. Results thus show that a $3^{\circ}$ frontal knee malalignment, though with moderate correlation, does not influence pressure distribution on plantar surface during gait. It is hence suggested that clinical evaluation should not be limited to static knee assessment, but rather include dynamic activities.

KEY wORDs: Biomechanics; Gait; Knee joint; Posture 


\section{INTRODUÇÃO}

O membro inferior pode ser considerado como uma cadeia cinética ${ }^{1-3} \mathrm{e}$, como tal, pressupõe-se que uma alteração biomecânica em um dos complexos articulares dessa cadeia pode influenciar negativamente a biomecânica e função dos demais complexos.

A literatura tem demonstrado que um moderado desalinhamento frontal do joelho piora o prognóstico de doenças degenerativas, como a osteoartrite ${ }^{4}$. Dependendo da orientação do desvio do joelho, se em valgo ou em varo, mesmo que de apenas dez graus ${ }^{5}$, as forças articulares tanto estática quanto dinâmica não mais homogeneamente distribuídas favoreceriam o surgimento de processos degenerativos nos compartimentos lateral ${ }^{5}$ e medial do joelho ${ }^{6}$, respectivamente. Pode-se inferir poir que uma alteração postural frontal no joelho comprometeria sobremaneira as estruturas articulares, levando ao desenvolvimento de lesões nesse segmento, o que conseqüentemente prejudicaria sua função.

De toda essa cadeia, o pé desempenha um papel de grande importância, uma vez que, sendo o segmento mais distal da extremidade inferior, é o responsável pela base de suporte do corpo. Qualquer alteração na superfície desse apoio poderia influenciar ou induzir estratégias motoras compensatórias que resultariam em mudanças ascendentes, como por exemplo no joeIho, o que poderia levar a alteração nas atividades da vida diária, tal como na marcha ${ }^{3}$.

Uma das proposições clínico-científicas descritas na literatura relativa à fisioterapia é um elo mecânico funcional entre o joelho e o complexo do tornozelo e pé $e^{7-10}$. Uma diminuição no ângulo frontal do joelho, decorrente de uma modificação nas articulações proximais do membro inferior (quadril), poderia influenciar a angulação do fêmur e/ou da tíbia e, secundariamente, o complexo do tornozelo e pé ${ }^{11,12}$. Em um sistema ascendente, uma movimentação anormal (maior ou menor) da articulação subtalar geralmente pode desencadear lesões no joelho, devido à influência mecânica entre essas duas articulações, por meio da conexão com a tíbia ${ }^{13}$.
A importância do complexo tornozelo e pé para a estabilidade mecânica da cadeia cinética dos membros inferiores é destacada por outros autores, já que ajustes motores nesse complexo são fundamentais para a execução das habilidades de locomoção ${ }^{13,14}$. Guichet et al. ${ }^{15}$ estabelecem que a função do membro inferior deve ser clinicamente avaliada em situações estáticas e dinâmicas, levando-se em conta, particularmente, a progressão e geometria de colocação do pé no solo, considerando sua influência substancial no alinhamento mecânico do membro inferior.

Jaarsma et al. ${ }^{2}$ encontraram forte e significativa associação entre o ângulo de progressão do pé (toe out angle) durante a marcha e a rotação lateral do fêmur, após redução de fratura por meio de haste intra-medular. Keenan et al. ${ }^{7}$ demonstraram, durante a marcha, uma associação entre o desalinhamento em valgo dos joelhos e o valgo do antepé de pacientes com doenças reumáticas. Os autores sugerem que esse tipo de desalinhamento de joelho levaria a uma maior força em pronação do complexo tornozelo e pé, particularmente sobre a articulação subtalar, o que resultaria em mudanças nos padrões da marcha. A sobrecarga medial em antepé durante a marcha induzida pelo desalinhamento em valgo de joelho também foi discutida por Sobel et al. ${ }^{9}$.

Partindo desses princípios, pode-se pressupor que um desalinhamento postural ascendente ou descendente nessa cadeia cinética inferior resultaria em alterações na dinâmica da marcha. Porém, a literatura ainda é deficitária na comprovação consistente da relação entre os desalinhamentos estáticos dos joelhos e as possíveis compensações ou alterações na dinâmica da locomoção de indivíduos assintomáticos, tanto que Poletto et al. ${ }^{16}$, por fotogrametria e eletrogoniometria, não encontraram correlação entre a postura estática dos joelhos e a variação angular dinâmica na marcha.

Mesmo assim, na prática fisioterapêutica, ao avaliar estaticamente os desalinhamentos posturais do joelho, ainda se infere que, quando as estruturas dessa articulação se encontram desalinhadas e em posições biomecânicas desfavoráveis, haverá desvantagem na execução da marcha.
Diante do exposto, o objetivo do presente estudo foi avaliar o efeito do alinhamento frontal estático do joelho na distribuição das pressões sobre a superfície das solas dos pés durante a marcha em adultos jovens assintomáticos.

\section{METODOLOGIA}

Foram estudados 44 indivíduos assintomáticos de ambos os sexos, com idade entre 18 e 40 anos. Foram excluídos indivíduos amputados, com presença de alteração neurológica periférica ou central, fratura de membros inferiores, que apresentaram algum quadro álgico nos últimos 6 meses ou que possuíam discrepância entre os membros inferiores maior do que $2 \mathrm{~cm}$. Os indivíduos assinaram um termo de consentimento livre e esclarecido formal; o estudo foi aprovado pelo Comitê de Ética e Pesquisa da Faculdade de Medicina da Universidade de São Paulo.

\section{Avaliação postural do joelho}

$\mathrm{O}$ indivíduo, trajando roupas de banho, foi posicionado ortostaticamente diante de uma parede não reflexiva a uma distância de $15 \mathrm{~cm}$ da mesma, com os pés descalços e um retângulo de etilvinil-acetado de $7,5 \mathrm{~cm}$ de largura entre os pés para manter uma distância padrão. Foram demarcadas, sempre pelo mesmo fisioterapeuta, as espinhas ilíacas ântero-superiores (EIAS) e os centros de ambas as patelas, utilizando-se etiquetas brancas de $0,9 \mathrm{~cm}$. Utilizou-se um fio de prumo afixado no teto e ao lado do sujeito para posterior calibração da imagem. A captura da imagem foi realizada por meio de uma câmera fotográfica digital (Sony) com resolução de 4.1 megapixels posicionada sobre um tripé em frente e perpendicular ao indivíduo fotografado, a uma distância de 2,5 m e uma altura de $0,75 \mathrm{~m}$ do solo, adequadamente centralizado e nivelado ${ }^{17}$.

Para avaliação das imagens e mensuração do ângulo frontal do joelho foi utilizado o programa para avaliação postural SAPO (v.0.63). Por meio deste, traçou-se uma reta da EIAS até o ponto médio bicondilar na altura da patela e daí até o ponto médio bimaleolar do tornozelo (Figura 1). Utilizou-se essa metodologia pois a espinha do platô tibial, 


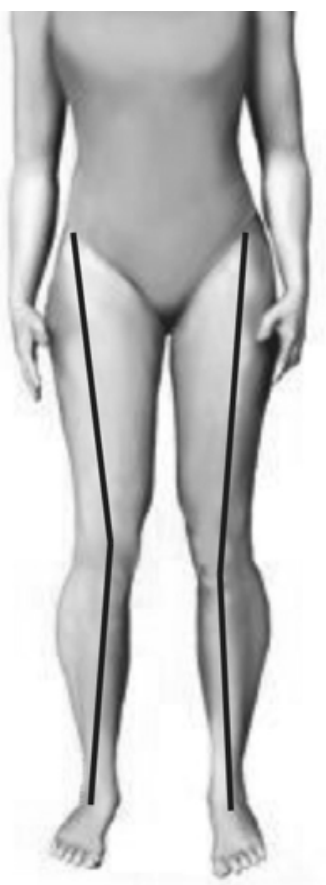

Figura 1 Ilustração da mensuração do ângulo frontal do joelho

considerada por Norkin e Levangie ${ }^{18}$ Como o centro articular do joelho e comumente visualizada por meio de exame radiográfico, não poderia ser estimada diretamente por imagens fotográficas.

Os joelhos foram classificados como: normais $\left(170^{\circ}\right.$ a $\left.175^{\circ}\right)$, varos $\left(>175^{\circ}\right) \mathrm{e}$ valgos $\left(<170^{\circ}\right)$, de acordo com Neumann ${ }^{19}$. Após a mensuração dos ângulos articulares dos sujeitos, avaliados estatisticamente, constatou-se, pela classificação adotada, apenas seis joelhos valgos (três sujeitos). Optou-se então por analisar apenas os casos de joelhos normais e varos, em um total de 41 sujeitos. Os indivíduos foram divididos em dois grupos, de acordo com o alinhamento frontal do joelho: grupo de joelhos normais (GJN) $-\mathrm{n}=18 ; 173,5 \pm 2,1^{\circ}$ de alinhamento frontal de joelho; $25 \pm 7$ anos, $56,2 \pm 11,0 \mathrm{~kg}, 167,0 \pm 8,8 \mathrm{~cm}$; e grupo de joelhos varos (GJVr) - n=23, $177,5 \pm 1,1^{\circ}$ de alinhamento frontal de joelho; $25 \pm 6$ anos, $58,6 \pm 14,9 \mathrm{~kg}$, $170,0 \pm 10,5 \mathrm{~cm}$.

\section{Avaliação da distribuição da pressão plantar}

Para mensuração da distribuição da pressão plantar durante a marcha, foram utilizadas palmilhas capacitivas do sistema Pedar X (Novel, Alemanha) compostas de 99 sensores com resolução de 1 sensor $/ \mathrm{cm}^{2}$. As palmilhas foram fixadas ao pé do indivíduo por meio de uma meia antiderrapante e conectadas a um acondicionador colocado dentro de uma mochila fixada e justaposta às costas dos sujeitos avaliados (Figura 2).

Os sujeitos caminharam por uma pista de borracha de $10 \mathrm{~m}$ com cadência auto-selecionada (aproximadamente 100 bpm). Os dados foram amostrados a $100 \mathrm{~Hz}$ e a transmissão dos dados para o computador foi feita via bluetooth para posterior análise. As variáveis da pressão plantar pico de pressão $(\mathrm{kPa})$, área de contato $\left(\mathrm{cm}^{2}\right)$, integral de pressão (kPa.s) e tempo de contato (ms) foram avaliadas em cinco áreas plantares: retropé lateral e medial, médio-pé e antepé medial e lateral. A aquisição, o processamento e a análise dos sinais foram feitos por meio de programas do sistema Novel (Novel Multiprojects) e a análise estatística foi realizada no programa Statistica v.8.

Após a confirmação dos pressupostos de normalidade, por meio do teste W de Shapiro Wilk e de homogeneidade de variâncias pelo teste de Levene, as quatro variáveis de pressão plantar foram comparadas entre os grupos por meio de quatro Anovas dois fatores ( $2 \times 5)$, tendo como fatores independentes os dois grupos e como medidas repetidas as cinco áreas do pé. Também se buscou investigar a correlação entre as medidas angulares do joelho e as variáveis da pressão plantar por meio da correlação de Pearson. Adotou-se um nível de significância de 5\%.

\section{RESULTADOS}

A descrição dos resultados da distribuição da pressão plantar está na Tabela 1. Os grupos mostraram-se estatisticamente semelhantes em todas as variáveis avaliadas em todas as áreas plantares.

O ângulo de alinhamento normal de joelhos apresentou moderada e significativa correlação com o tempo de contato no antepé lateral $(r=0,40 ; p=0,018)$ e médio-pé ( $r=0,48 ; p=0,003)$. Já o ângulo de alinhamento varo dos joelhos

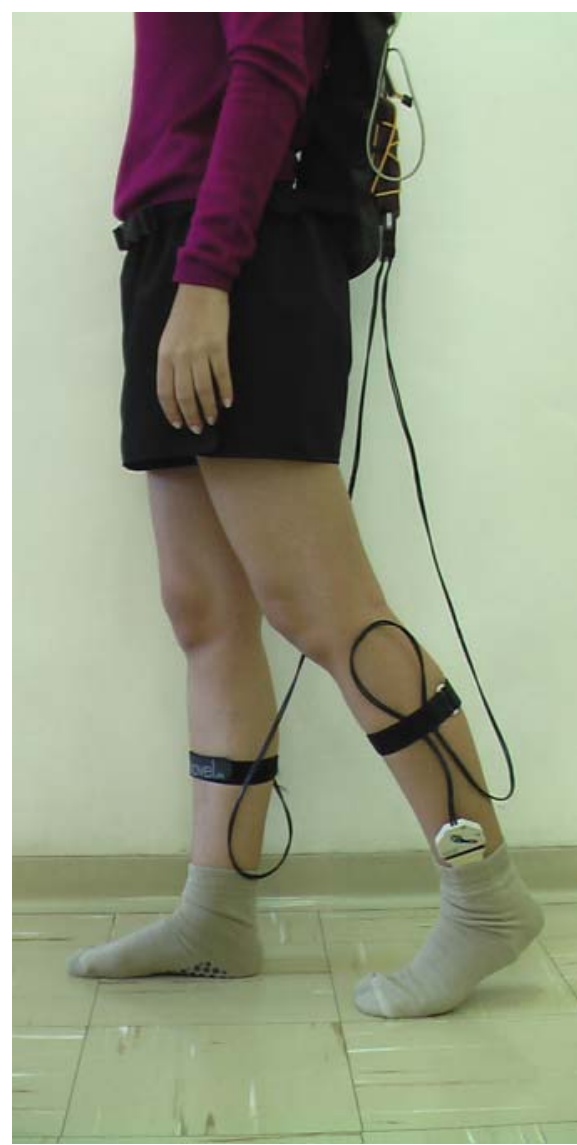

Figura 2 Sujeito instrumentado com as palmilhas fixadas ao pé por meio de meias antiderrapantes e a mochila para acondicionamento dos equipamentos

apresentou mais correlação com a distribuição da pressão plantar, ou seja, as variáveis área de contato, tempo de contato e integral da pressão também no antepé e médio-pé correlacionaram-se moderada e significativamente com os ângulos em varo do joelho $(0,34<r>$ 0,$40 ; \mathrm{p}<0,02$ ) (Tabela 2).

\section{DISCUSSÃO}

O objetivo do presente estudo foi avaliar o efeito do alinhamento frontal estático do joelho na distribuição da pressão plantar durante a marcha de adultos jovens assintomáticos. Os resultados mostraram que em sujeitos sem sintomas e queixas por conta de seu alinhamento frontal estático do joelho não há influencia dessa postura na distribuição de cargas na superfície plantar, muito embora quan- 
Tabela 1 Área de contato (cm2), pico de pressão (kPa), tempo de contato $(\mathrm{ms}) \mathrm{e}$ integral da pressão ( $\mathrm{kPa} . \mathrm{s}$ ) nas cinco áreas plantares (média \pm desvio padrão) dos grupos com joelhos normais (GJN) e com joelhos varos (GJVr) e valores de $p$ da comparação entre os grupos

\begin{tabular}{|c|c|c|c|c|}
\hline & Áreas plantares & GJN (n=18) & GJVr $(n=23)$ & $p$ \\
\hline \multirow{5}{*}{$\begin{array}{l}\text { Área de } \\
\text { contato }\left(\mathrm{cm}^{2}\right)\end{array}$} & Retropé medial & $19,59 \pm 3,57$ & $19,23 \pm 3,93$ & 0,990 \\
\hline & Retropé lateral & $9,64 \pm 3,83$ & $10,90 \pm 4,14$ & 0,970 \\
\hline & Médio-pé & $18,09 \pm 7,44$ & $17,08 \pm 6,49$ & 0,995 \\
\hline & Antepé medial & $35,40 \pm 3,39$ & $35,92 \pm 5,04$ & 0,999 \\
\hline & Antepé lateral & $24,92 \pm 3,93$ & $25,46 \pm 4,24$ & 0,999 \\
\hline \multirow{5}{*}{$\begin{array}{l}\text { Pico de } \\
\text { pressão }(\mathrm{kPa})\end{array}$} & Retropé medial & $355,44 \pm 87,33$ & $344,61 \pm 70,25$ & 0,999 \\
\hline & Retropé lateral & $248,44 \pm 68,43$ & $291,02 \pm 82,45$ & 0,198 \\
\hline & Médio-pé & $93,60 \pm 37,72$ & $90,23 \pm 28,14$ & 1,000 \\
\hline & Antepé medial & $403,42 \pm 79,72$ & $367,13 \pm 74,61$ & 0,422 \\
\hline & Antepé lateral & $275,53 \pm 80,52$ & $308,04 \pm 82,22$ & 0,587 \\
\hline \multirow{5}{*}{$\begin{array}{l}\text { Tempo de } \\
\text { contato (ms) }\end{array}$} & Retropé medial & $538,86 \pm 88,56$ & $530,70 \pm 92,69$ & 0,999 \\
\hline & Retropé lateral & $457,79 \pm 102,79$ & $485,21 \pm 107,22$ & 0,863 \\
\hline & Médio-pé & $435,08 \pm 93,93$ & $436,56 \pm 72,88$ & 1,000 \\
\hline & Antepé medial & $568,76 \pm 45,44$ & $584,19 \pm 44,08$ & 0,996 \\
\hline & Antepé lateral & $568,89 \pm 43,09$ & $591,99 \pm 43,70$ & 0,948 \\
\hline \multirow{5}{*}{$\begin{array}{l}\text { Integral da } \\
\text { pressão } \\
(\mathrm{kPa} . \mathrm{s})\end{array}$} & Retropé medial & $81,77 \pm 16,77$ & $81,45 \pm 18,25$ & 1,000 \\
\hline & Retropé lateral & $56,65 \pm 17,78$ & $68,57 \pm 17,70$ & 0,122 \\
\hline & Médio-pé & $25,70 \pm 11,60$ & $25,20 \pm 8,28$ & 1,000 \\
\hline & Antepé medial & $96,63 \pm 16,40$ & $97,88 \pm 20,43$ & 1,000 \\
\hline & Antepé lateral & $75,80 \pm 24,99$ & $89,02 \pm 23,06$ & 0,136 \\
\hline
\end{tabular}

Tabela 2 Correlação (coeficiente $r$ de Pearson e valor de p) entre o ãngulo de alinhamento do joelho e variáveis da distribuição da pressão plantar

\begin{tabular}{lrrc}
\hline Variáveis da pressão plantar & Alinh. & $r$ & $p$ \\
\hline Tempo de contato médio-pé & JN & 0,48 & 0,003 \\
Tempo de contato antepé lateral & JN & 0,40 & 0,018 \\
Área de contato médio-pé & JV & 0,33 & 0,022 \\
Área de contato antepé medial & JV & 0,35 & 0,018 \\
Tempo de contato médio-pé & JV & 0,40 & 0,006 \\
Tempo de contato antepé medial & JV & 0,34 & 0,021 \\
Tempo de contato antepé lateral & JV & 0,40 & 0,005 \\
Integral da pressão antepé lateral & JV & 0,34 & 0,018
\end{tabular}

Alinh. = alinhamento dos joelhos; JN = joelhos normais; $\mathrm{JV}=$ joelhos varos

to mais varo o ângulo frontal de joelho, maiores as correlações com essa distribuição de pressão na marcha.

Estes resultados podem ser explicados por algumas hipóteses: (i) ausência de associação entre as medidas estáticas (fotogrametria) e dinâmicas (distribuição de pressão plantar) utilizadas como metodologia; (ii) características inerentes à amostra estudada; e (iii) va- riabilidade característica da distribuição da pressão plantar durante a marcha.

A primeira hipótese que justificaria os resultados do presente estudo é que de fato não haja influência da postura estática frontal de joelho sobre as respostas dinâmicas do pé durante a marcha, e não necessariamente a não-existência de associação entre os segmentos joelho e pé. Corroborando parcialmente essa hipótese, Poletto et al. ${ }^{16}$ também verificaram a ausência de correlação entre a postura estática dos joelhos, avaliada por fotogrametria, e a dinâmica na marcha, avaliada por eletrogoniometria. Contudo, os autores não incluíram medidas que relacionassem o alinhamento do joelho com a funcionalidade do pé, tal como a distribuição das forças no pé decorrente desse alinhamento.
Por outro lado, Shohei et al..$^{20}$, ao utilizarem somente mensurações dinâmicas (cinemetria e pressão plantar), encontraram associação entre o alinhamento do joelho e do pé. A tarefa motora analisada foi a de aterrissagem unipodal a partir de uma caixa de $30 \mathrm{~cm}$ de altura. Isso a torna uma tarefa que gera maior sobrecarga à base de sustentação do membro inferior, o que, segundo os autores, leva a um aumento da pressão na região do arco longitudinal do pé, devido a alterações mais acentuadas no alinhamento dinâmico dos membros inferiores. Porém, durante a aterrissagem, grande parte da sobrecarga corporal é imposta predominantemente sobre uma única região do pé - nesse caso, o médio-pé. Já durante a marcha, a tarefa avaliada no presente estudo, a sobrecarga é distribuída ao longo de toda a superfície plantar durante o processo de rolamento do pé. Diante disso, acredita-se que os discretos desalinhamentos estáticos do joelho observados na amostra estudada não tenham sido suficientes para alterar a distribuição da pressão plantar durante a marcha, ao ponto de resultar em diferenças entre os grupos.

Silva et al. ${ }^{21}$, em um estudo com iniciantes de atividade física em academia, também observaram uma associação entre as posturas do joelho e do pé, mais precisamente, entre o valgismo do joelho e desabamento do arco plantar. Contudo, cabe ressaltar que esses resultados foram obtidos por inspeção visual durante a marcha e na postura estática. Além disso, Silva et al. ${ }^{21}$ não descrevem se as correlações discutidas em seu estudo foram obtidas somente em uma condição, ou seja, nas observações estáticas ou dinâmicas.

A segunda hipótese que explicaria os resultados do presente estudo seria a própria característica da amostra. A mesma foi composta de adultos jovens assintomáticos, não atletas, sem queixas, sem sobrepeso e apresentando discretos desalinhamentos estáticos dos membros inferiores (de $169^{\circ}$ a $180^{\circ}$ ). Diante disso, pouca ou nenhuma alteração poderia ser esperada sobre a distribuição da pressão durante a marcha, uma vez que grande parte dos joelhos estava inserida dentro dos parâmetros da normalidade $-170^{\circ}$ a $175^{\circ}$, de acordo com 
Neumann ${ }^{19}$. Vale ressaltar que, de um total de 88 joelhos avaliados, somente seis foram classificados como valgos de acordo com os parâmetros descritos por Neumann ${ }^{19}$, para quem o valgo fisiológico recebe a classificação "normal" (daí a opção por avaliar somente dois grupos, de joelhos varos e normais).

Diante desses resultados, acredita-se que os indivíduos avaliados estão bem adaptados à marcha, que é uma habilidade motora diária e habitual. Talvez se poderia esperar que as respostas biomecânicas fossem alteradas em tarefas motoras mais desafiadoras em termos de controle e de exigência muscular, como correr, saltar, subir e descer escadas. Isso certamente levaria a uma exacerbação do alinhamento frontal do joelho, semeIhante ao que foi observado no estudo de Shohei et al. ${ }^{20}$.
Contudo, não se pode predizer que esse resultado se reproduza em amostras compostas por indivíduos susceptíveis a maiores sobrecargas dos membros inferiores, como é o caso de atletas, obesos ou mesmo em indivíduos sintomáticos ou, ainda, com importantes desalinhamentos frontais de joelhos.

Por fim, com base na terceira hipótese, as não-diferenças significativas entre os grupos podem ter sido atribuídas à razoável variabilidade da distribuição da pressão plantar (coeficiente de variação entre 35 e 50\%), além do efeito do erro do tipo b provocado pela amostra reduzida deste estudo. Acredita-se que, com aumento da amostra estudada, a variabilidade poderia ter sido atenuada e diferenças entre os grupos poderiam ficar mais evidentes.

\section{CONCLUSÃO}

O alinhamento frontal estático do joelho não influenciou a distribuição das pressões plantares durante a marcha de adultos jovens assintomáticos. Devido à moderada correlação encontrada entre o alinhamento frontal estático do joeIho e as respostas biomecânicas dinâmicas sugere-se que essa relação seja ponderada, e que a avaliação clínica fisioterapêutica estática não seja feita isoladamente e sim, avaliada em conjunto com atividades dinâmicas, como na marcha, corrida ou no subir e descer escadas. Em situações de maior acentuação do alinhamento frontal do joelho, no caso de varo ou valgo extremos, acredita-se que alguma alteração da pressão na superfície plantar possa ser observada.

\section{REFERENNCIAS}

1 Guskiewicz KM, Perrin DH. Research and clinical applications of assessing balance. J Sport Rehabil. 1996;5(1):45-63.

2 Jaarsmaa RL, Ongkiehonga BF, Grünebergb C, Verdonschota N, Duysensb J, van Kampena A. Compensation for rotational malalignment after intramedullary nailing for femoral shaft fractures: an analysis by plantar pressure measurements during gait. Injury. 2004;35(12):1270-8.

3 Cote KP, Brunet ME, Gansneder BM, Shultz SJ. Effects of pronated and supinated foot postures on static and dynamic postural stability. J Athl Train. 2005;40(1):41-6.

4 Felson DT, Goggins J, Niu J, Zhang Y, Hunter DJ. The effect of body weight on progression of knee osteoarthritis is dependent on alignment. Arthritis Rheum. 2004;50(12):3904-9.

5 Johnson RJ, Ettlinger CF, Shealy JE, Meader C. Impact of super sidecut skis on the epidemiology of skiing injuries. Sportsverletz Sportschaden. 1997;11:150-2.

6 Sharma L, Song J, Felson DT, Cahue S, Shamiyeh E, Dunlop DD. The role of knee alignment in disease progression and functional decline in knee osteoarthritis. JAMA. 2001;286(2):188-95.

7 Keenan MA, Peabody T, Gronley J, Perry J. Valgus deformities of the feet and characteristics of gait in patients who have rheumatoid arthritis. J Bone Joint Surg. 1991;73(2):237-47.
8 McLauren CAN, Wootton JR, Heath PD, Wynn Jones $\mathrm{CH}$. Pes planus after tibial osteotomy. Foot Ankle. 1989;9(6):300-3.

9 Sobel M, Stern SH, Manoli A II, Bohne WHD. The association of posterior tibial tendon insufficiency with valgus osteoarthritis of the knee. Am J Knee Surg. 1992;5:59-64.

10 Teitge RA, MacMahon E. Osteoarthritis of the knee: concepts behind osteotomy. Semin Arthroplasty. 1996;7(2):114-23.

11 Verderi E. Programa de educação postural. São Paulo: Phort; 2001.

12 Cailliet, R. Síndromes dolorosas: joelho, dor e incapacidade. São Paulo: Manole; 1987.

13 Williams DS, McClay IS, Hamil J. Arch structure and injury patterns in runners. Clin Biomech (Bristol, Avon). 2001;16:341-7.

14 Belchior ACG, Arakaki JC, Bevilaqua-Grossi D, Reis FA, Carvalho PTC. Effects in the Q angle measurement with maximal voluntary isometric contraction of the quadriceps muscle. Rev Bras Med Esporte. 2006;12(1):5e-8e.

15 Guichet JM, Javed A, Russell J, Saleh M. Effect of the foot on the mechanical alignment of the lower limbs. Clin Orthop Relat Res. 2003;415:193-201. 


\section{Referências (cont.)}

16 Poletto PR, Sato TO, Carnaz L, Lobo DA, Costa PH, Gil Coury HJC. Indivíduos que apresentam diferença estática entre os joelhos também apresentam diferença durante a marcha? Rev Bras Fisioter. 2007;11(1):43-8.

17 Iunes DH, Castro FA, Salgado HS, Moura IC, Oliveira AS, Bevilaqua-Grossi D. Confiabilidade inter e intraexaminadores e repetibilidade da avaliação postural pela fotogrametria. Rev Bras Fisioter. 2005;9(3):249-55.

18 Norkin CC, Levangie PK. Joint: structure \& function; a comprehensive analysis. Philadelphia: Davis; 1992.

19 Neumann DA. Kinesiology of the musculoskeletal system: foundations for physical rehabilitation. Philadelphia: Mosby; 2002.
20 Shohei M, Shumpei M, Nao U, Naoyuki M, Issei O, Masahiro T, et al. The relation between medial longitudinal arch of foot and the knee alignment at single-legged landing maneuver. In: 12th Annual Congress of the ECSS; 11-14 July, Jyyaskyla, Finland; 2007. Disponível em: https://ecss2007.cc. jyu.fi/ schedule/proceedings/pdf/1786.pdf.

21 Silva AS, Souza MSC, Morais ER, Silva JMF, Canuto OS, Athayde RA, et al. Prevalência de alterações posturais para prescrição do programa de exercícios em academias de ginástica - PB. Rev Saude.com. 2005;1(2):124-33. 